\title{
The Structure of Carbon Encapsulated NiFe Nanoparticles
}

\author{
Wendy Teunissen,* Frank M . F. de Groot, *,1 John G eus,* O dile Stephan, $\dagger$ \\ $M$ arcel Tence, $\dagger$ and Christian Colliex $\dagger \dagger$ \\ *D ebye Institute, D epartment of I norganic Chemistry and Catalysis, U trecht U niversity, Sorbonnelaan 16, 3584 CA, U trecht, The N etherlands; \\ $\dagger \mathrm{L}$ aboratoire de Physique des Solides, Bâtiment 510, CN R S U M R 8502, F 91405 O rsay, France; and $¥ \mathrm{~L}$ aboratoire A imé Cotton, \\ Bâtiment 505, CNRS UPR 3321, F 91405 O rsay, France
}

R eceived A pril 26, 2001; revised J une 26, 2001; accepted A ugust 4, 2001

\begin{abstract}
Carbon encapsulated NiFe nanoparticles ( $\mathrm{NiFe@C)}$ have been prepared by high-temperature methane encapsulation of the bare bimetallic particles on alumina. High-resolution transmission electron microscopy pictures show that about 6-nm thick carbon layers encapsulate 10-20-nm diameter NiFe nanoparticles. The $\mathrm{NiFe}$ nanoparticles are shown to be single-crystalline and no carbide is found at the $\mathrm{NiFe}-\mathrm{C}$ interface. This is confirmed by the electron energy-loss spectroscopy (EELS) measurements that in addition show that both $\mathrm{Ni}$ and Fe have a zero (metal) valence and that only graphite is present. EELS also shows that the nickel-to-iron ratio is exactly unity for all particles studied. Metallic Pd nanoparticles with a diameter of 1-2 $\mathrm{nm}$ can be anchored on the carbon layers, which creates a Pd/NiF e@C type of catalyst that could be used for liquid phase reactions. The EELS analysis reveals that part of the nanoparticles present are not Pd but other oxidic carbon encapsulated nanoparticles. (c) 2001 Academic Press
\end{abstract}

Key Words: carbon encapsulation; TE M; EE LS; magnetic separation.

\section{INTRODUCTION}

A major goal in catalysis research is the development of small solid bodies that can be suspended in and extracted from the liquid phase. These bodiesneed to be small to overcome the transport limitations in the liquid phase. Bodies smaller than a few $\mu \mathrm{m}$ cannot be separated by filtration or centrifugation. Magnetic separation offers a route to use particles of about $50 \mathrm{~nm}$, but requires these nanoparticles to have a sufficiently high saturation magnetization, while redispersion asks for magnetic softness. NiFe and CoFe bimetallic systems are satisfactory candidates from the magnetic point of view. For the particles to be used as catalytic support material, they must be inert and resistant to the conditions during catalytic reactions. B are metallic nanoparticles certainly do not fulfill these requirements and a solution is the embedding of the bimetallic nanoparticles in carbon sheets.

\footnotetext{
${ }^{1}$ To whom correspondence should be addressed. Fax: ++31 302511027 E-mail: f.m.f.degroot@chem.uu.nl.
}

Carbon encapsulation has attracted much attention after the discoveries of fullerenes, single-walled carbon tubes, and related systems $(1,2)$. M ultiwalled carbon fibers, also known as carbon filaments, have a long history, ever since the 1889 patent on the growth of carbon filaments by metal crucibles (3). Throughout the twentieth century the nucleation and growth of carbon fibers has been an important field of research, mainly because of the destructive nature of carbon fibers on reactor walls $(4,5)$. The knowledge of the prevention of carbon fiber growth automatically yielded new insights on the optimization of carbon fiber growth modes and, closely related, on the growth of encapsulated metal particles (5).

\section{EXPERIMENTAL}

Carbon encapsulated NiFe particles (NiFe@C) have been prepared by (a) growing $\mathrm{NiFe}$ metal particles on $\gamma$ alumina, (b) encapsulating these NiFe metal particles in a methane flow, followed by removal of alumina and also any incompletely covered particles with an acid treatment, and (c) having Pd nanoparticles anchored on the outside of the carbon encapsulated particles. Compared to the arc discharge method (1,6-9), as well as other methods (10-12), this catalytic route to the production of carbon encapsulated nanoparticles offers a much more detailed control of, for example, the alloy composition. In addition, scaleup is expected to be easier.

(a) The NiFe particles are prepared by depositing sodium nitroferricyanide $\left(\mathrm{Na}_{2} \mathrm{Fe}(\mathrm{CN})_{5} \mathrm{NO}\right)$ and nickel nitrate $\mathrm{Ni}\left(\mathrm{NO}_{3}\right)_{2}$ precursors, which have a nickel-to-iron ratio of $1: 1$. The aqueous iron cyanide solution is injected at a rate of $1 \mathrm{ml} / \mathrm{min}$ into a vigorously stirred suspension of $\gamma$-alumina in an aqueous solution of nickel nitrate and kept at $\mathrm{pH}$ 5. The NiFe loaded alumina was filtered, washed, and dried at room temperature in a vacuum for $24 \mathrm{~h}$. The $425-$ $850-\mu \mathrm{m}$ sieve fraction was calcined in a $95 \mathrm{ml} / \mathrm{min}$ flow of $20 \%$ oxygen in helium at $573 \mathrm{~K}$ for $3 \mathrm{~h}$. This gives $\mathrm{NiFe-}$ oxide particles on alumina. This treatment is followed by a reduction in a $100 \mathrm{ml} / \mathrm{min}$ flow of $20 \%$ hydrogen in argon 
at $700^{\circ} \mathrm{C}$ for $2 \mathrm{~h}$. This gives about $20 \mathrm{wt} \%$ bare $\mathrm{NiFe}$ alloy particles on $\gamma$-alumina $(13,14)$.

(b) The encapsulation procedure is based on the large body of knowledge developed for the growth of carbon fibers (5). The alloy particles were brought to $998 \mathrm{~K}$ under flowing hydrogen, exposed to a $100 \mathrm{ml} / \mathrm{min}$ flow of $20 \%$ methane in argon at $998 \mathrm{~K}$ for $1 \mathrm{~h}$ and cooled to room temperature in a flow of argon. This yields carbon encapsulated $\mathrm{NiFe}$ particles as well as partly uncovered alloy particles. To isolate the encapsulated NiFe particles, $200 \mathrm{mg}$ of alumina was dissolved by boiling the sample with $50-\mathrm{ml}$ concentrated hydrochloric acid for $30 \mathrm{~min} .200 \mathrm{ml}$ of cold demineralized water was added and the material was filtered, washed, and dried in air at room temperature. The acid treatment has removed all $\mathrm{NiFe}$ particles with porous carbon layers, in this way excluding the presence of nickeliron alloy particles in not fully closed shells. The relative yield of the NiFe particles prepared in this manner is $68 \%$ (14).

(c) To prepare NiFe@C supported palladium catalysts, we used incipient wetness impregnation with $\mathrm{PdCl}_{2}$ dissolved in hydrochloric acid. It turned out from previous research on carbon fibers that no further activation procedure is required for anchoring the palladium complex on the carbon $(15,16)$. The pore volume (voids between the spherical NiFe@C particles) of the material was measured to be $0.9 \mathrm{ml} / \mathrm{g}$. To prepare a catalyst with a Pd loading of 2.5 wt\%, NiFe@C was placed in an impregnation flask and brought under static vacuum. The calculated amount of the Pd solution was injected into the flask and kept under static vacuum for $15 \mathrm{~min}$. N ext, an argon flow was passed over at a temperature of $333 \mathrm{~K}$ for $1 \mathrm{~h}$. The $\mathrm{Pd}$ complex anchored to the carbon layers was converted to metallic palladium upon reduction in hydrogen. Samples of Pd/NiFe@C were placed in a reactor in a gas flow of $10 \% \mathrm{H}_{2}$ in $\mathrm{Ar}$, raising the temperature with $1.5 \mathrm{~K} / \mathrm{min}$ to $473 \mathrm{~K}$.

\section{RESULTS AND DISCUSSION}

The geometric and electronic structure of NiFe@C particles has been characterized with transmission electron microscopy (TEM) and electron energy-loss spectroscopy (EELS) using a scanning TEM. The TE M images were acquired with a Topcon 002B electron microscope operating at $200 \mathrm{kV}$, equipped with $\mathrm{a} \mathrm{LaB}_{6}$ filament, and providing a point resolution of about $0.2 \mathrm{~nm}$. Samples were prepared by suspending the solid in ethanol under ultrasonic vibration. One or two drops of the thus prepared suspension were brought onto a punched carbon film on a copper grid. TheE E LS data were recorded with a scanning transmission electron microscope VG HB 501, equipped with a fieldemission source and a parallel G atan 666 E E LS spectrometer. This instrument produces EELS spectra with a typical $0.7-\mathrm{eV}$ resolution recorded from subnanometer areas

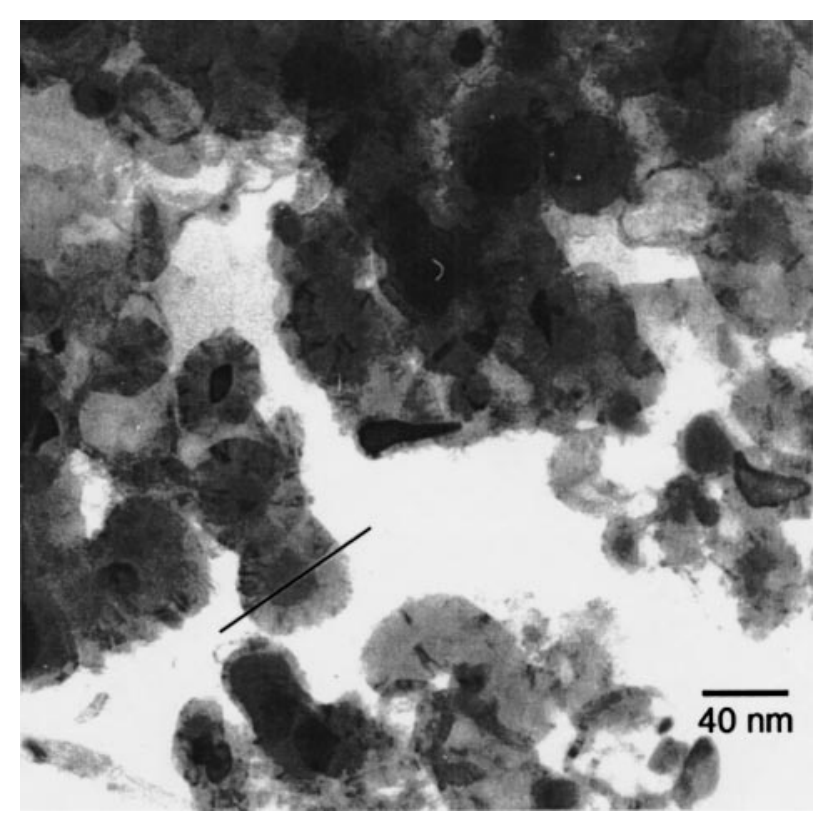

FIG. 1. TEM image of carbon coated nickel-iron particles. The line indicates the probe position during the EE LS analyses.

$(17,18)$. In the line-spectrum mode, spectra are recorded while ramping the probe with given steps across the specimen. A cquisition times required for achieving a satisfactory signal-to-noise level on core edges are on the order of 100 ms- $1 \mathrm{~s}$. A checking of the materials after E E LS measurements indicated that there was no radiation-induced structural damage.

A TEM picture of the particles is shown in Fig. 1. The metallic core is encapsulated in polyhedral concentric graphitic shells with a varying number of layers. Figure 2 shows a high-resolution photo of the lattices of the metallic core and the surrounding carbon. From this figure the lattice distance found for the N iFe core is $0.21 \mathrm{~nm}$, in agreement with the (111) lattice dimension of metallic $\mathrm{NiFe}$. For graphite we measured a lattice distance of $0.34 \mathrm{~nm}$, close to its (002) lattice. A II particles studied showed only one orientation of the $\mathrm{NiFe}$ lattice, without grain boundaries. This indicates that the particles measured are single crystals. A s far as detectable from the images, no dissimilarity in the lattice distances are observed at the interface of the NiFe metal core and the carbon layers. This indicates that no carbidic phase is present, an observation that will be confirmed by the EELS measurements.

A n EELS spectrum, taken at a probe position on the $\mathrm{NiFe}$ metal core is represented in Fig. 3. The electron, energy-loss fine structure of the carbon $\mathrm{K}$ edge at $280 \mathrm{eV}$, the iron $L_{2,3}$ edge at $710 \mathrm{eV}$, and nickel $L_{2,3}$ edge at $850 \mathrm{eV}$ are clearly visible. The iron and nickel edges are much weaker than the carbon $\mathrm{K}$ edge and they have been multiplied by 8.0 in Fig. 3. The spectral shapes of the EELS core edges provide chemical information on the nature of the elements 


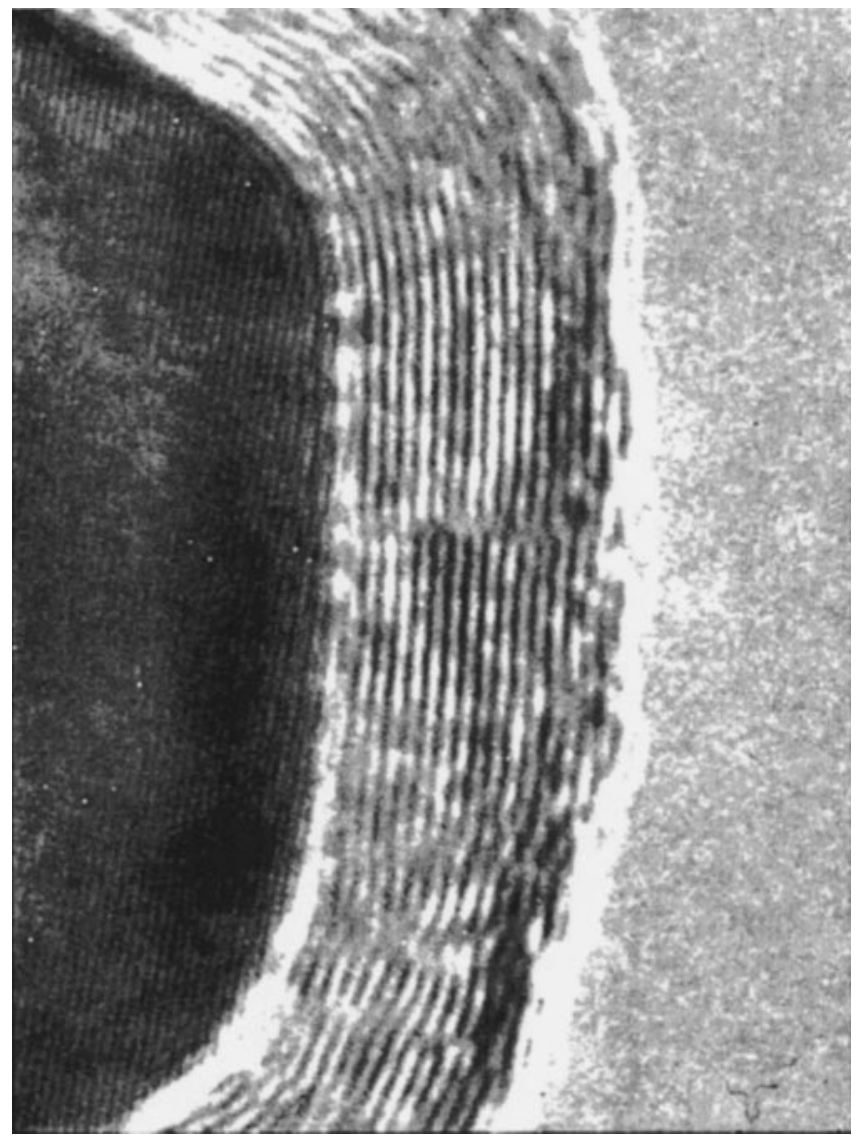

FIG. 2. H igh-resolution TE M image of the $\mathrm{NiFe}$ core and the surrounding graphite layers. The graphite layer thickness is approximately $6 \mathrm{~nm}$.

$(19,20)$. The nickel and iron $L_{2,3}$ spectra (shown with better statistics in Fig. 5) indicate metallic nickel respectively iron (21), while the carbon $\mathrm{K}$ edge is reminiscent of graphitic carbon.

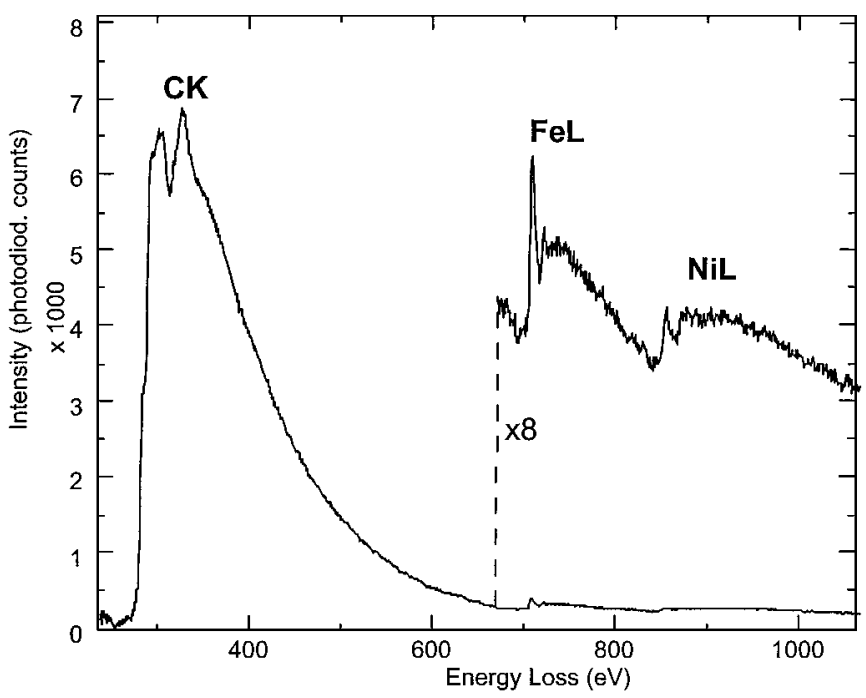

FIG. 3. E lectron energy loss fine structure of the carbon $K$ edge and the iron and nickel $L$ edge. The spectrum is taken at one probe position.

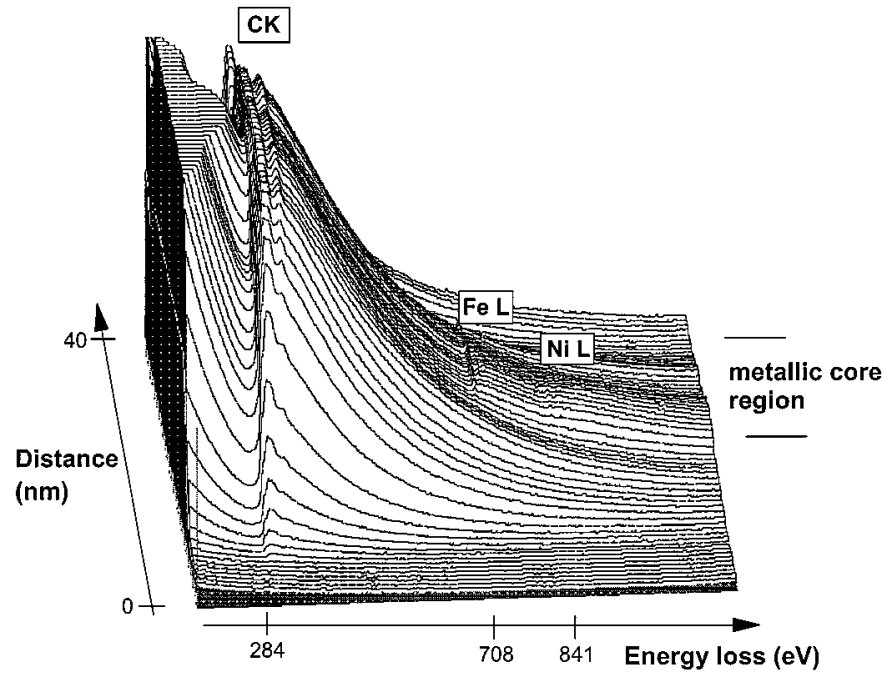

FIG. 4. $2 D$ plot of a line spectrum ( 64 spectra) as the probe beam scans along a line of approximately $40 \mathrm{~nm}$ from vacuum through a $35-\mathrm{nm}$ $\mathrm{NiFe@C} \mathrm{particle.} \mathrm{Horizontal} \mathrm{scans} \mathrm{represent} \mathrm{the} \mathrm{energy-loss} \mathrm{values.}$

Figure 4 represents the $2 \mathrm{D}$ plot of a sequence of spectra taken across a particle of about $35 \mathrm{~nm}$ with a metallic core of about $12 \mathrm{~nm}$. The path was chosen through the center of the particle as indicated in Fig. 1. The sequence of 64 spectra was recorded with a $0.5-\mathrm{nm}$ probe, a $0.6-\mathrm{nm}$ pixel increment, and an acquisition time of $1 \mathrm{~s}$ per pixel. This amounts to a total path length of $40 \mathrm{~nm}$ within a total acquisition time of $64 \mathrm{~s}$. To obtain the nickel-to-iron ratio within the alloy particle, the cross sections of the carbon $K$ edge, nickel $L$ edge, and iron $L$ edge were estimated for every E E LS spectrum. Figure 5 shows part of one E E L S spectrum. The region used for background subtraction is indicated before the iron and nickel edges and the resulting background is given as the

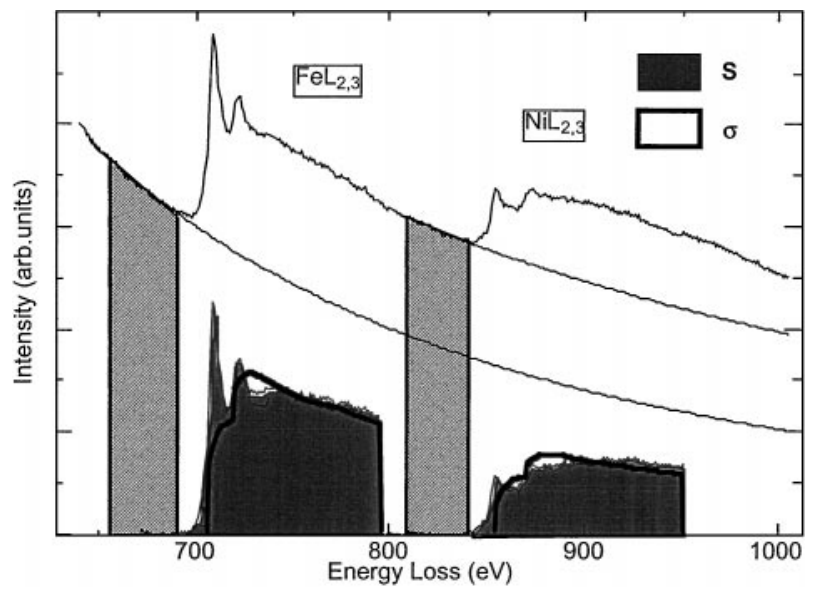

FIG. 5. $L_{2,3}$ edges for iron and nickel recorded from carbon encapsulated particles. A Iso shown are the two characteristic signals after background subtraction, the energy window for integration of the characteristic signals and calculated ionization cross sections, and the calculated atomic cross sections calculated in a $\mathrm{H}$ artree-Slater model. 


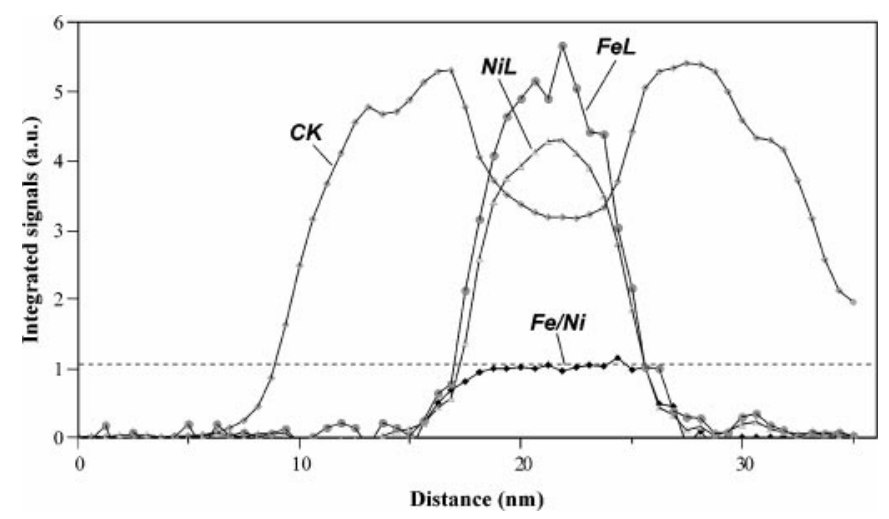

FIG. 6. The normalized intensity variations of the carbon $K$, nickel $L_{2,3}$, and iron $L_{2,3}$ edges as the beam is scanned across an encapsulated $\mathrm{NiFe}$ particle.

thin lines. The characteristic signals after background subtraction are shown with filled surfaces, indicating also the energy window for integration of the characteristic signals and the calculated ionization cross sections (22). In order to improve the statistics and limit potential sample damage, we did measure the E E L S spectra over the core level region only; i.e., the plasmon region was not detected in parallel. We note that thishas the disadvantage that for thick samples it is not possible to correct the intensity profile for multiple scattering events.

The results of the analysishave been summarized in Fig. 6. No elements other than carbon, nickel, and iron were detected. A s the beam scans across the particle, the intensity of the energy-loss peaks corresponding with the $\mathrm{K}$ edge in graphite (window: 280-325 eV) increases corresponding with the increasing number of carbon atoms in the column that the beam encounters, exhibits a drop in the center due to the internal hollow space, and follows the same pattern on the other side. The shape of the profile essentially shows a cylindrical geometry (23), which is in agreement with a small slice in the center of a spherical particle projected in a two-dimensional way. A distortion from a perfect hollow sphere can be observed in the small dip around the position at $14 \mathrm{~nm}$. This indicates that the graphitic layer deviates from a perfect sphere. The intensity of the energy loss from the $L_{2,3}$ edges of nickel and iron correlates well with the position of the central core of the particle and the peaking of intensity in the center clearly indicates that the alloyed nickel and iron is present as a solid sphere and is not just wetting the inside surface. A n elemental quantification using tabulated atomic $2 p$ ionization crosssections (24) gives a constant ratio of nickel and iron very close to unity, in agreement with the adjusted nickel-to-iron ratio in the cyanide complex used during preparation, namely $\mathrm{NiFe}(\mathrm{CN})_{5} \mathrm{NO}$. A similar result was found for all six investigated particles. In summary, the shape of the different curves in Fig. 6 confirms the approximately spherical geometry of the particle, with a hollow core for carbon, filled with a solid core with a nickel-to-iron ratio of unity.

No spectral variations were found across the iron and nickel $L_{2,3}$ edges in the particle core and also at the interface with graphite. The iron and nickel spectral shapes (cf. Fig. 5) indicate that both are present in their metallic state (21). The carbon $\mathrm{K}$ edge indicated in Fig. 3 corresponds to graphite and shows no indication for the presence of any carbidic species in the filled portion of the particle. The high-temperature synthesis of the surrounding graphite layers seems to result in nonsegregated, $\mathrm{NiFe}$ alloy particles encapsulated by closed shells of pure graphite with the absence of carbides at the interface. H owever, the comment must be made that a carbidic phase formed during synthesis, not finally resulting in nonporous surrounding graphitic layers, will be removed during treatment in hydrochloric acid. This result confirms the HRTEM images (cf. Fig. 2), where also no indication was found for a carbidic phase.

\section{A nchoring of Pd Particles}

A TEM micrograph of a NiFe@C-supported palladium catalyst after reduction is represented in Fig. 7. Clearly visible are the small particles on the external edge of the carbon layers. M ost of these particles turned out to be metallic $\mathrm{Pd}$, but as will be discussed below other types of small particles were found from the E E LS analysis. The large particles are the ferromagnetic NiFe cores of the support. A part from small particles on the outside of the NiFe@C particles, also small particles are visible at other positions in the sample,

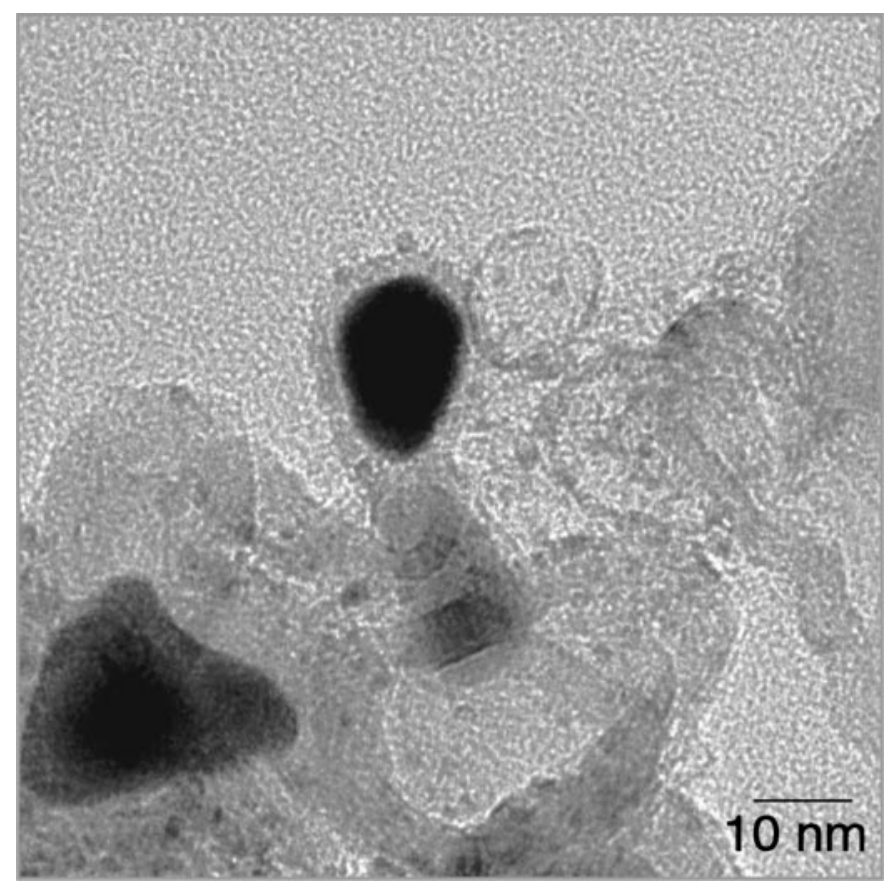

FIG. 7. TEM images of palladium on NiFe@C, prepared via impregnation. 


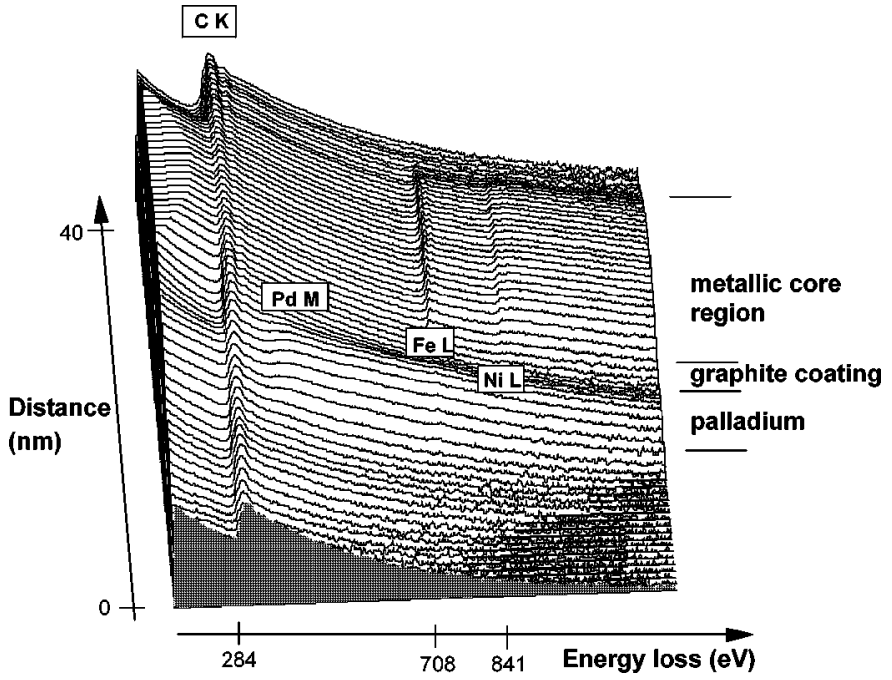

FIG . 8. 2D plot of a line spectrum along a line of approximately $40 \mathrm{~nm}$ from vacuum through a $60-\mathrm{nm} \mathrm{Pd} / \mathrm{N}$ iFe@C particle. H orizontal scans represent the energy-loss values.

for example, on the empty sphere to the right of the NiFe@C particle.

Figure 8 shows the 2D plot of a sequence of spectra taken halfway across a particle of about $60 \mathrm{~nm}$ with a metallic core of about $40 \mathrm{~nm}$. The sequence was recorded in the same manner as Fig. 4. In addition to the carbon $\mathrm{K}$ edge at $280 \mathrm{eV}$, the iron $L_{2,3}$ edge at $710 \mathrm{eV}$, and the nickel $L_{2,3}$ edge at $850 \mathrm{eV}$, the $\mathrm{Pd} \mathrm{M} \mathrm{M}_{2,3}$ edge at $532 \mathrm{eV}$ can be observed. Figure 9 displays the profile of the relative intensities of $\mathrm{Pd}, \mathrm{C}, \mathrm{Ni}$, and Fe calculated from the line scan shown in Fig. 8 and using the same procedure as described above for the NiFe@C particles. The carbon profile of Fig. 9 agrees with an almost perfect hollow spherical carbon particle. Palladium is located on the external edge of the particle, anchored to the carbon layers. A particle with a diameter of approximately $5 \mathrm{~nm}$ is found in Fig. 9. The spectral shape of the Pd $M_{2,3}$

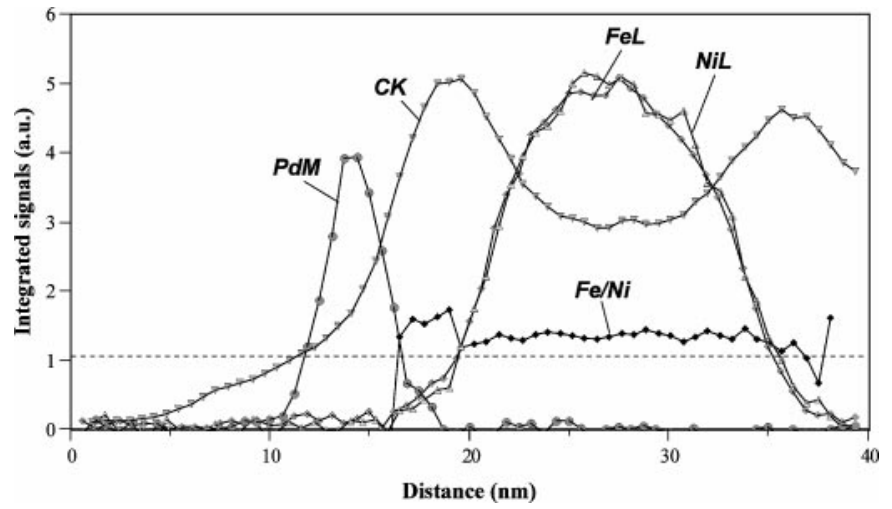

FIG . 9. The variation of intensity of carbon $\mathrm{K}$, palladium $\mathrm{M}_{2,3}$, nickel $L_{2,3}$, and iron $L_{2,3}$ edges as the beam is scanned across a Pd/NiFe@C particle. edge shows that $\mathrm{Pd}$ is in its metallic state. However, we should mention that the detection of a small amount of oxygen is complicated due to the presence of the $\mathrm{Pd} \mathrm{M}_{3}$ edge in the $O \mathrm{~K}$ energy range. We were not able to detect any deviation of the $\mathrm{Pd}$ or $\mathrm{C}$ spectra at the $\mathrm{Pd} / \mathrm{C}$ interface, but we cannot rule out detection limitations due to the very small interface area. The iron-to-nickel ratio is constant throughout the particle, but deviates from the adjusted $1: 1$ ratio.

A II particles with a N iFe core less than $12 \mathrm{~nm}$ in diameter show exactly the 1:1 ratio. Therefore we believe that this deviation is due to the incorrect background modeling and the consequent incorrect evaluation of the characteristic absorption signals. A s indicated above, we did not measure the plasmon region of the E E LS spectrum simultaneously, which excludes the elimination of multiple scattering effects. The $\mathrm{Ni}$ : Fe ratio in Fig. 9 is still found to be constant over the core of particle. This ratio seems to increase in the carbon region between the NiFe core and palladium nanoparticle. H owever, as the absolute $\mathrm{N}$ i and Fe intensities decrease in this region, the uncertainty in the ratio increases and the determined value is not reliable enough to draw a firm conclusion.

A systematic scan on all small particles that are observable with EELS in a certain area showed that only about half of these particles consisted of Pd. Some of the small particles we examined turned out to be iron oxide or nickeliron oxide, fully covered with graphite, where in some cases also aluminium is present. In Fig. 10 the E E L S spectrum is shown of the analysis of a nickel-iron oxide particle, obtained by using the gain variation routine in the derivative mode. The particles found can originate from the interlayer between the metal and alumina support, which often is hard to reduce. With the formation of encapsulating graphite

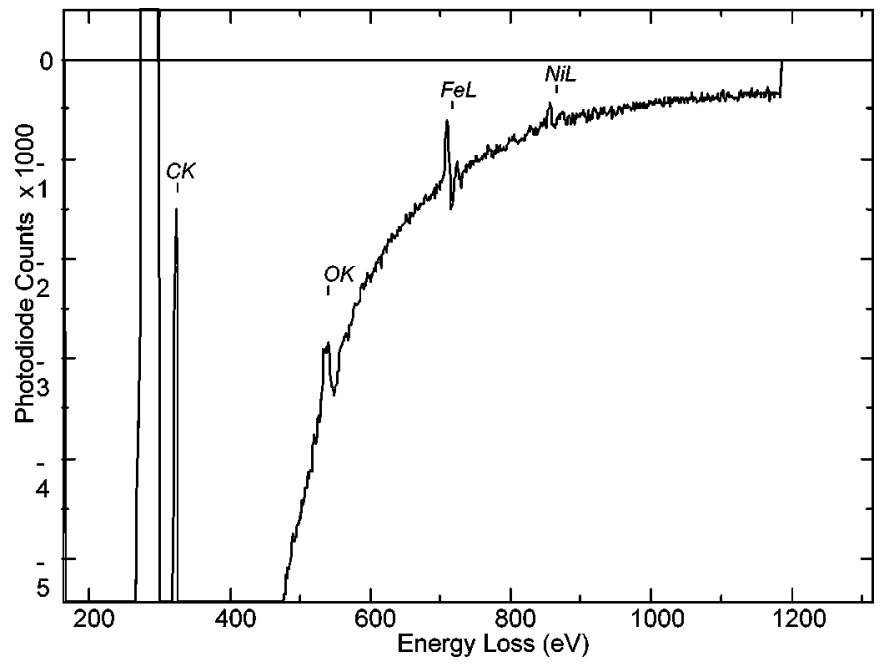

FIG. 10. Electron energy-loss fine structure of the carbon $K$ edge, the oxygen $\mathrm{K}$ edge, and the iron and nickel $\mathrm{L}_{2,3}$ edge. The spectrum is obtained using the gain variation routine, recorded in the derivative mode. 
layers the interaction between the alloy particles and support has to be overcome. We suggest that the interface be broken up, leaving some particles in the oxidic phase which become fully encapsulated. These iron oxide, or nickel-iron oxide, encapsulated particles will also be present in the sample where no palladium is anchored to the NiFe@C particles. H owever, in a TE M picture a palladium nanoparticle of $2 \mathrm{~nm}$ will be better visible than a 2-nm N iFe-oxide particle. It is only with an E E LS analysis that these nanoparticles reveal their elemental nature.

\section{CONCLUSIONS}

It can be concluded that TE M-E E L S provides a detailed picture of the nature of the carbon encapsulated metal particles. The E E LS intensity profiles reveal the relative ratio of the elements present. It should be noted that EELS intensity profiles have a spatial resolution of approximately $0.5 \mathrm{~nm}$, which is intrinsically much better than the resolution that can be obtained from an E DX elemental analysis. The EE LS elemental analysis is quantitatively correct for thin samples. For samples with a thickness larger than about $30 \mathrm{~nm}, \mathrm{E}$ E L S can also yield quantitatively correct values if the values are corrected for multiple scattering events. The high spatial resolution of the E E L S spectra has been used to study potential chemical interface effects. These effects were not observed with the resolution of $0.5 \mathrm{~nm}$.

The NiFe core is found to be metallic and singlecrystalline with a lattice distance equal to bulk NiFe and with exactly a $1: 1 \mathrm{~N} \mathrm{i}$ : Fe ratio. The diameter of the particles varies between 10 and $30 \mathrm{~nm}$. The particle is encapsulated by graphitic planes with an interplanar distance equal to bulk graphite. The graphite forms a hollow sphere around the NiFe nanoparticle and no carbidic interface between the metal core and the graphite is visible.

We conclude that the preparation method for anchoring small Pd particles to carbon encapsulated NiFe particles is very effective. This keeps alive promises for the development of encapsulated metal particles as catalyst support materials that can be suspended in, magnetically extracted from, and redispersed in the liquid phase.

$\mathrm{H}$ alf of the small nanoparticles observed in TE $M$ are related to carbon encapsulated oxides, which is a result that could only be obtained from the EELS analysis. It would be very hard to distinguish such oxidic particles from the $\mathrm{Pd}$ particles using TEM only.

\section{ACKNOWLEDGMENTS}

This work was carried out as part of the I nnovation O riented $\mathrm{R}$ esearch Programme on Catalysis (IOP Catalysis IK A 94080) sponsored by the $N$ etherlands $M$ inistry of $E$ conomic $A$ ffairs. The research of $F d G$ has been supported by the $\mathrm{N}$ etherlands R esearch Combination Catalysis.

\section{REFERENCES}

1. K ratschmer, W., L amb, L. D., Fostiropoulos, K ., and H uffman, D. R ., N ature 347, 354 (1990).

2. Iijima, S., N ature 354, 56 (1991).

3. Hughes, T. V., and Chambers, C. R ., U.S. Patent 405, 480 (1889).

4. R ostrup-Nielsen, J. R., J. Catal. 85, 31 (1984).

5. de Jong, K. P., and G eus, J. W., Chem. Eng. R ev. Sci. E ng. 42, 481 (2000).

6. D ravid, V. P., H ost, J. J., Teng, M. H., Elliot, B., H wang, J., Johnson, D. L., M ason, T. O., and Weertman, J. R ., N ature 374, 602 (1995).

7. Scott, J. H. J., and Majetlich, S. A., Phys. Rev. B 52, 12,564 (1995).

8. D ong, X. L., Z hang, Z. D., J in, S. R ., and K im, B. K., J. A ppl. P hys. 86, 6701 (1999).

9. Sun, X., G utierrez, A ., Y acaman, M . J., D ong, X . L., and J in, S., M at. Sci. Eng. A 286, 157 (2000).

10. Nolan, P. E., Lynch, D. C., and Cutler, A. H., J. Phys. Chem. B 102, 4165 (1998).

11. Nolan, P. E., Lynch, D. C., and Cutler, A. H., Carbon 31, 817 (1996).

12. H arris, P. J. F., and Tsang, S. C., Chem. P hys. L ett. 293, 53 (1998).

13. Teunissen, W., and Geus, J. W., Stud. Surf. Sci. Catal. 121, 185 (1998).

14. Teunissen, W., Bol, A. A ., and Geus, J. W., Catal. Today 48, 329 (1999); Wendy Teunissen, Ph.D. thesis. U trecht U niversity, U trecht, The N etherlands, 2000.

15. Hoogenraad, M. S., van Leeuwarden, R. A. G. M. M., van Breda V riesman, G. J. B., B roersma, A ., van Dillen, A . J., and G eus, J. W., Stud. Surf. Sci. Catal. 91, 263 (1995).

16. Mojet, B. L., Hoogenraad, M. S., van Dillen, A. J., Geus, J. W., and Koningsberger, D. C., J. Chem. Soc. Faraday Trans. 93, 4371 (1997).

17. Jeanguillaume, C., and Colliex, C., Ultramicroscopy 28, 252 (1989).

18. Colliex, C., Tencé, M ., L efèvre, E ., M ory, C., G u, H ., B ouchet, D., and Jeanguillaume, C., M ikrochim. A cta 71, 114 (1994).

19. Colliex, C., in "NATO A SI Series, Series B: Physics" (P. S. Bagus, G. Pacchioni, and F. Parmigiani, Eds.), Vol. 345, p. 213, Colliex, C., J. E lectron. M icrosc. 45, 44 (1996).

20. de G root, F. M. F., J. E lec. Spectrosc. 67, 529 (1994).

21. Fink, J., M üller-H einzerling, Th., Scheerer, B., Speier, W., H illebrecht, F. U., Fuggle, J. C., Zaanen, J., and Sawatzky, G. A ., Phys. Rev. B 32, 4899 (1985).

22. E gerton, R . F., in "E lectron E nergy-L oss Spectroscopy in the E lectron M icroscope." Plenum, New York, 1986.

23. Suenaga, K., Colliex, C., D emoncy, N., L oiseau, A ., Pascard, H ., and Williame, F., Science 278, 653 (1997).

24. Leapman, R. D., R ez, P., and M ayers, D. F., J. Chem. Phys. 72, 1232 (1980). 